\title{
Comparaisons interlaboratoires de comptage de l'activité des émetteurs alpha et bêta dans des eaux à différentes charges salines
}

\author{
G. Granier ${ }^{1}$, P. Nardoux ${ }^{2}$ et D. Roudil ${ }^{1}$ \\ ${ }^{1}$ CEA/MARCOULE, DEN/DRCP/CETAMA, B.P. 17171, 30207 Bagnols sur Ceze, France. \\ 2 AREVA Mines SEPA, B.P. 71, 87250 Bessines, France.
}

Reçu le 29 janvier 2015 - Accepté le 26 mai 2015

\begin{abstract}
Résumé - La CETAMA a organisé 3 essais de comparaisons interlaboratoires depuis 2011 afin d'évaluer la capacité des laboratoires à déterminer l'activité globale des radionucléides émetteurs alpha et bêta des eaux représentatives de matrices environnementales. La natures des radionucléides (naturels et artificiels) et la variation de la charge saline de la solution, de faiblement minéralisé jusqu'à celle de l'eau de mer, sont les variables prises en compte. Les modalités de ces essais ont été définies au sein du groupe de travail «Analyse de l'eau» dans le but de résoudre certaines difficultés rencontrées lors de la réalisation de la mesure des activités alpha et bêta globales. L'identification des causes de l'écart constaté entre les valeurs obtenues lors des comptages et la somme des activités de chacun des radionucléides pris séparément en est la raison principale. Par ailleurs ces essais permettent aux laboratoires accrédités ou en cours d'accréditation de réaliser un exercice préparatoire en amont de ceux organisés par l'IRSN pour la délivrance des agréments. À partir des valeurs de référence des différents radionucléides contenus dans les échantillons, déterminées par un laboratoire primaire de métrologie, les moyennes sur les résultats obtenus par les laboratoires sont calculées et comparées à celles-ci. L'exploitation des résultats a permis de mettre en évidence la difficulté d'obtenir une valeur de référence de comptage pour la détermination d'une activité globale alpha et béta. La prédominance des conditions opératoires et des paramètres de mesure dans l'obtention du résultat final en est la raison majeure. La démarche poursuivie pour la détermination de la valeur assignée de comptage assortie de son incertitude, établie à partir de documents de référence (normes, guides...) est présentée de manière détaillée. La synthèse des différents essais a permis de dégager des recommandations, notamment en termes d'étalonnage et de préparation de la source, qui permettent d'améliorer sensiblement la justesse et la fidélité de la méthode.
\end{abstract}

\begin{abstract}
Synthesis of a Round Robin about measurement of gross alpha and beta in various waters. Since 2011 the CETAMA (Analytical Methods Committee) has organized 3 Round-Robin tests successively to determine the capability of laboratories to measure gross alpha and beta in various waters. Different parameters are considered such as the origin of radionuclides (natural and artificial) and the variation of the salt content (from a low mineralized solution to highly mineralized water such as seawater). Technical specifications are defined within the working group "Water analysis" in order to solve some problems observed in measurement of gross alpha and beta activity concentrations. The objective is to identify the causes of a discrepancy between the values obtained from the total counts and the sum of the reference values of each radionuclide alone. These Round-Robin tests allow accredited laboratories (or nearly accredited) to train in order to obtain accepted results in French regulatory proficiency tests organized by the IRSN. From the reference values of the various radionuclides (delivered by a primary metrology laboratory) contained in the samples, the averages of the results obtained by the laboratories are calculated and compared. Synthesis of the results highlighted the difficulty in obtaining reference values for gross alpha and gross beta activity. A method is proposed, established from standard and official guidelines based on robust calculation, to determine these assigned values with their associated uncertainty. Feedback from the various interlaboratory comparisons led to suggesting recommendations, particularly in terms of calibration and sample preparation, which allow a significant improvement in the accuracy (trueness and precision) of the method.
\end{abstract}

Keywords: gross alpha / gross beta / water / counting efficiency / uncertainties

^ guy.granier@cea.fr 


\section{Introduction}

Depuis sa création en 1961 la Commission d'ETAblissement des Méthodes d'Analyse (CETAMA), unité du CEA (Commissariat à l'énergie atomique et aux énergies alternatives) a pour fonction principale l'amélioration de la qualité des résultats de mesures. Elle assume cette mission grâce à l'animation de groupes de travail thématiques. Ses actions s'articulent autour de la rédaction de méthodes, des projets de normes et ouvrages sur la mesure et la diffusion des informations scientifiques au travers la tenue de conférences. L'élaboration de matériaux de référence et l'organisation d'essais interlaboratoires complètent ces dispositions. Cela répond à un besoin spécifique des analyses en milieu nucléaire, qui nécessitent une qualité de mesure qui couvre un domaine de concentration ou d'activité s'étendant sur plusieurs ordres de grandeur.

Les essais de comparaisons interlaboratoires permettent une évaluation de l'aptitude de laboratoires à réaliser une caractérisation donnée, adaptée à cette grande diversité de situation. Plus largement, les attendus de ces essais sont multiples. Ils se révèlent être un moyen efficace de faire converger plus rapidement les modes opératoires vers une méthode beaucoup plus robuste que les pratiques isolées, souvent spécifiques au traitement d'un cas particulier. Cela nécessite toutefois un important travail de synthèse qui implique les participants « Rien de ce qui se passe à l'intérieur d'une centrale nucléaire ne doit avoir de conséquences à l'extérieur et vice versa ». Le législateur fonde sa doctrine sur ce principe (Bizet et Boog, 2014) convaincu du bien-fondé de la mesure comme moyen efficace de diagnostic.

La loi sur la transparence nucléaire dite loi $\mathrm{TSN}^{1}$ fixe un cadre juridique à l'évaluation des risques radiologiques et à la surveillance de l'impact des activités liées à l'industrie nucléaire sur l'environnement. L'État confie à l'Autorité de Sureté Nucléaire ${ }^{2}$ (ASN) la mission de contrôle de l'état radiologique de l'environnement aussi bien à l'intérieur et proximité des installations nucléaires que sur l'ensemble du territoire. Les principaux acteurs de la surveillance de la radioactivité dans l'environnement en France sont tenus de respecter ces directives (ASN, 2015).

C'est le réseau national de mesures ${ }^{3}$ (RNM) de la radioactivité de l'environnement, instauré par le code de la santé publique $^{4}$, qui accomplit la mission de centraliser les informations sur la radioactivité de l'environnement en France. Il assure sa fonction de contrôle en établissant une cartographie de la radioactivité présente sur l'ensemble du territoire français.

Les textes règlementaires, édités par Euratom ${ }^{5}$ (Communauté européenne de l'énergie atomique; EURATOM, 2013)

\footnotetext{
${ }^{1}$ Loi du 13 juin 2006 relative à la transparence et à la sécurité en matière nucléaire : «Toute personne a le droit, dans les conditions définies par la présente loi et les décrets pris pour son application, d'être informée sur les risques liés aux activités nucléaires et leur impact sur la santé et la sécurité des personnes ainsi que sur l'environnement, et sur les rejets d'effluents des installations ».

${ }^{2}$ http://www.asn.fr/

3 http://www.mesure-radioactivite.fr/public/

${ }^{4}$ Code de la santé publique articles R 1333-11 et R 1333-11-1.

${ }^{5}$ La directive générale $N^{\circ}$ 96/29/Euratom du Conseil du 13 mai 1996, s'applique à toutes les activités dans lesquelles interviennent
}

définissent le droit européen en la matière et sont ensuite déclinés en droit local. Leur doctrine se base sur le principe qu'une dose indicative de radiation résultant de l'ingestion de radionucléides présents dans une eau destinée à la consommation humaine ne dépasse pas un certain seuil. La correspondance entre la dose de rayonnement absorbé, qui se mesure en Gray et l'activité de substances radioactives qui se mesure en Bq, est fondée sur des études publiées par l'OMS (WHO, 2011) sur la base de recommandations émises par la CIPR $^{6}$ (Commission Internationale de Protection Radiologique).

En France les indicateurs et les valeurs guide de contrôle de la qualité radiologique des eaux sont précisées dans une circulaire émanant du Ministère de la santé (2007).

Le premier niveau d'investigation analytique est la mesure de l'activité alpha globale qui est un indicateur de présence de radionucléides émetteurs alpha et celle de l'activité bêta globale résiduelle qui est un indicateur de présence de radionucléides émetteurs bêta autres que le ${ }^{40} \mathrm{~K}$. Basé sur le principe qu'une dose infime de radiation peut avoir des effets notables sur la santé, les calculs partent de l'hypothèse qu'une consommation annuelle moyenne de 730 litres d'eau par habitant ne doit pas conduire à une dose efficace de radiation supérieure à $0,1 \mathrm{mSv}$. De ce fait elle prescrit depuis le $25 / 12 / 2003$, sur toutes les eaux de distribution, à l'exclusion des eaux minérales, la mesure de paramètres radiologiques tritium, alpha total et bêta total. Si le comptage alpha total dépasse $0,1 \mathrm{~Bq} . \mathrm{L}^{-1}$ et le comptage bêta résiduel $1 \mathrm{~Bq} . \mathrm{L}^{-1}$ des investigations complémentaires sont préconisées pour identifier les radionucléides émetteurs. Il s'agit de rechercher parmi les radionucléides naturels; les isotopes 234, et 238 de l'uranium, le ${ }^{226} \mathrm{Ra}$, le ${ }^{228} \mathrm{Ra}$, le ${ }^{210} \mathrm{Po}$ et le ${ }^{210} \mathrm{~Pb}$. Dans les eaux thermales et minérales, une recherche des isotopes 228, 230 et 232 du thorium peut compléter la précédente.

La maitrise de ces mesures est absolument capitale car elles constituent le premier niveau d'investigation pour l'évaluation des risques et conditionne la mise en œuvre de programmes analytiques spécifiques. Disposer de données est une nécessité absolue, mais la confiance dans celles-ci doit être optimale pour que l'évaluation globale soit la plus représentative possible des risques encourus. La maitrise du processus de mesure, y compris dans son volet prélèvement, est une préoccupation essentielle du législateur qui a le souci de hisser toujours plus haut le niveau de surveillance et d'information.

Le RNM met à disposition du public, via son site internet, toutes les mesures de radioactivité réalisées par les différents organismes qui les produisent. Cela requiert de disposer de données de confiance pour évaluer d'une manière la plus fiable possible les doses dues aux rayonnements ionisants auxquels la population est exposée. Seuls les laboratoires agréés sont habilités à transmettre leurs résultats au RNM. Cet agrément est délivré par l'ASN sous réserve que ceux-ci respectent les exigences de la norme NF EN ISO/CEI 17025 (ISO, 2005a)

des rayonnements ionisants, et qui fixe les normes de base concernant la protection sanitaire de la population et des travailleurs contre les dangers résultant des rayonnements ionisants. Elle va être remplacée par la directive 2013/59 Euratom du 5 décembre 2013 fixant les normes de base en radioprotection qui sera transposée dans tous les États membres.

6 http://www.icrp.org/ 
et satisfasse aux essais de comparaisons interlaboratoires organisés périodiquement par l'Institut de Radioprotection et de Sureté Nucléaire (IRSN) ${ }^{7}$. Ces différents types d'essais sont programmés par type de matrice environnementale et par catégorie de mesures radioactives. Ils sont définis par décision de l'ASN (2008). L'IRSN est accrédité par le COFRAC ${ }^{8}$ en qualité d'organisateur d'essais de comparaisons interlaboratoires selon la norme NF EN ISO/CEI 17025 (ISO, 2005a).

L'étude s'articule autour de 3 comparaisons interlaboratoires (CIL) de mesure des activités alpha et bêta globales dans les eaux, réalisés de 2010 à 2013 et dont les objectifs majeurs étaient de :

- permettre aux laboratoires participants de vérifier leur aptitude à effectuer un dosage de ces radionucléides selon la norme NF ISO 10704 (ISO, 2010a);

- appréhender la dispersion des résultats, aux niveaux d'activité testés, sur des matrices naturelles ou artificielles en faisant varier leur charge saline pour pouvoir ensuite les comparer aux résultats obtenus sur une eau à faible minéralisation;

- permettre aux laboratoires accrédités ou en cours d'accréditation, dans le cadre de l'agrément délivré par l'ASN, de réaliser un exercice préparatoire en amont des essais interlaboratoires proposés par IRSN.

Le constat de difficultés analytiques, de dispersion de résultats, voire de biais ont conduit à ordonnancer ces essais successifs avec chaque fois des différences dans la composition chimique et radiochimique des solutions.

Le présent document détaille les objectifs de chaque CIL, son protocole expérimental et propose une synthèse.

\section{Présentation des essais}

Quoique la mesure des activités alpha globale et bêta globale dans des eaux naturelles couvre une multitude de singularités chaque essai proposait des échantillons de natures différentes. Dans un souci de représentativité de situations les plus courantes il s'y trouvait des radionucléides naturels issus des chaînes de l'uranium et du thorium mais également des artificiels provenant d'activités industrielles.

Afin de discriminer au mieux les facteurs qui influent sur l'exactitude du résultat nous avons choisi :

- des solutions contenant uniquement des radionucléides naturels tels que l'uranium pour les émetteurs alphas et $1 e^{40} \mathrm{~K}$ pour les émetteurs béta;

- des solutions contenant des radionucléides artificiels, tels que les isotopes du plutonium pour les émetteurs alphas et le ${ }^{90} \mathrm{Sr}$ pour les émetteurs béta;

- une composition des deux propositions précédentes, contenant à la fois des radionucléides naturels et artificiels.

L'impact de la charge saline, y compris avec l'eau de mer a permis d'estimer son influence sur les résultats de comptage.

\footnotetext{
7 www.irsn.fr

8 http://www.cofrac.fr
}

Le principe retenu pour ces comparaisons interlaboratoires est d'évaluer l'aptitude des laboratoires à effectuer les mesures, en comparant leurs résultats obtenus avec une valeur de référence.

La principale conclusion de l'exploitation des résultats du premier essai a été la difficulté d'obtenir une valeur de référence pour les mesures d'activités alpha et bêta globales. En effet, comme le stipule la norme NF ISO 10704 (ISO, 2010a) dans son paragraphe 4 (principe) : «Les déterminations des activités alpha et bêta globales ne sont pas des déterminations absolues de la radioactivité alpha et bêta de l'échantillon, mais sont des déterminations relatives à des émetteurs alpha ou bêta spécifiques qui constituent les sources étalons de référence.»

Les conditions opératoires et les paramètres de mesure sont prédominants dans l'obtention du résultat final et propres à chaque utilisateur. Les activités alpha et bêta globale n'étant pas accessibles au sens métrologique du terme, il est toutefois possible d'attribuer une valeur consensuelle à partir des résultats d'une comparaison interlaboratoires. Cette valeur, communément appelée «valeur assignée » doit être assortie d'une incertitude.

La démarche s'est poursuivie par la mise en œuvre d'une méthodologie qui permette d'obtenir une valeur assignée de comptage à partir des résultats obtenus par les laboratoires participants.

\section{Nature des solutions des trois essais $(1,2$ et 3)}

Les laboratoires ont reçu pour le premier essai quatre solutions acidifiées à l'acide nitrique $3 \mathrm{M}$ :

A-1 Eau naturelle dopée en uranium et ${ }^{40} \mathrm{~K}$ à un niveau d'activité voisin de 70 Bq. $L^{-1}$;

B-1 Eau naturelle dopée en ${ }^{239} \mathrm{Pu}+{ }^{90} \mathrm{Sr}$ à un niveau d'activité d'environ $1 \mathrm{~Bq} . \mathrm{L}^{-1}$;

C-1 Eau naturelle dopée en ${ }^{239} \mathrm{Pu}+{ }^{90} \mathrm{Sr}$, uranium et ${ }^{40} \mathrm{~K}$ à un niveau d'activité de l'ordre de $100 \mathrm{~Bq} . \mathrm{L}^{-1}$;

D-1 Eau naturelle dopée en ${ }^{239} \mathrm{Pu}+{ }^{90} \mathrm{Sr}$, uranium et ${ }^{40} \mathrm{~K}$ avec une charge saline proche de 3 g.. - $^{-1}$ d'une activité de l'ordre de $90 \mathrm{~Bq} \cdot \mathrm{L}^{-1}$.

Les échantillons du deuxième essai sont composés de trois solutions acidifiées à l'acide nitrique $1 \mathrm{M}$ contenant :

A-2 Radionucléides émetteurs $\alpha, \beta$ et $\gamma\left({ }^{241} \mathrm{Am},{ }^{90} \mathrm{Sr}-{ }^{90} \mathrm{Y}\right.$, ${ }^{134} \mathrm{Cs}$ ) d'activité égale à $2 \mathrm{~Bq} . \mathrm{L}^{-1}$ en solution nitrate de calcium à $1 \mathrm{~g} . \mathrm{L}^{-1}$;

B-2 Radionucléides émetteurs $\alpha$, $\beta$ et $\gamma\left({ }^{241} \mathrm{Am},{ }^{90} \mathrm{Sr}^{-90} \mathrm{Y}\right.$, ${ }^{134} \mathrm{Cs}$ ) d'activité égale à $2 \mathrm{~Bq} . \mathrm{L}^{-1}$ en solution nitrate de strontium à $1 \mathrm{~g} . \mathrm{L}^{-1}$;

C-2 Radionucléides émetteurs $\alpha, \beta$ et $\gamma\left({ }^{241} \mathrm{Am},{ }^{90} \mathrm{Sr}-{ }^{90} \mathrm{Y}\right.$, ${ }^{134} \mathrm{Cs}$ ) d'activité égale à $2 \mathrm{~Bq} . \mathrm{L}^{-1}$ en solution nitrate de sodium à 1 g.L. $\mathrm{L}^{-1}$.

Les solutions du troisième circuit sont :

A-3 Radionucléides émetteurs $\alpha, \beta\left({ }^{239} \mathrm{Pu},{ }^{90} \mathrm{Sr}-{ }^{90} \mathrm{Y}\right)$, d'activité comprise entre 0,1 et 10 Bq.L $\mathrm{L}^{-1}$ en matrice de type eau acidifiée à l'acide nitrique $3 \mathrm{M}$; 
Tableau 1. Nature des solutions et charge saline.

Nature of solutions and saline concentration.

\begin{tabular}{lcccc}
\hline $\mathbf{N}^{\circ}$ & Matrice initiale & Famille Radionucléides & Radionucléide & Charge saline en g.L $\mathbf{L}^{-1}$ \\
\hline $\mathrm{A}-1$ & Eau naturelle & Naturel & $\mathrm{U}$ et ${ }^{40} \mathrm{~K}$ & 1,3 \\
$\mathrm{~B}-1$ & Eau naturelle & Artificiel & ${ }^{239} \mathrm{Pu}+{ }^{90} \mathrm{Sr}$ & 0,8 \\
$\mathrm{C}-1$ & Eau naturelle & Artificiel + Naturel & ${ }^{239} \mathrm{Pu}+{ }^{90} \mathrm{Sr}, \mathrm{U}$ et ${ }^{40} \mathrm{~K}$ & 1,4 \\
$\mathrm{D}-1$ & Eau naturelle & Artificiel + Naturel & ${ }^{239} \mathrm{Pu}+{ }^{90} \mathrm{Sr}, \mathrm{U}$ et ${ }^{40} \mathrm{~K}$ & $2,8 \mathrm{~g}$ \\
$\mathrm{~A}-2$ & Eau naturelle & Artificiel & ${ }^{241} \mathrm{Am},{ }^{90} \mathrm{Sr}-{ }^{90} \mathrm{Y},{ }^{134} \mathrm{Cs}$ & $1\left(\mathrm{CaNO}_{3}\right)$ \\
$\mathrm{B}-2$ & Eau naturelle & Artificiel & ${ }^{241} \mathrm{Am},{ }^{90} \mathrm{Sr}-{ }^{90} \mathrm{Y},{ }^{134} \mathrm{Cs}$ & $1\left(\mathrm{SrNO}_{3}\right)$ \\
$\mathrm{C}-2$ & Eau naturelle & Artificiel & ${ }^{241} \mathrm{Am},{ }^{90} \mathrm{Sr}-{ }^{90} \mathrm{Y},{ }^{134} \mathrm{Cs}$ & $1\left(\mathrm{NaNO}_{3}\right)$ \\
$\mathrm{A}-3$ & Eau naturelle & Artificiel & ${ }^{239} \mathrm{Pu},{ }^{90} \mathrm{Sr}-{ }^{90} \mathrm{Y}$, & 0,8 \\
$\mathrm{~B}-3$ & Eau de mer synthétique & Artificiel & ${ }^{239} \mathrm{Pu},{ }^{90} \mathrm{Sr}-{ }^{90} \mathrm{Y}$, & 35 \\
$\mathrm{C}-3$ & Eau de mer naturelle & Artificiel & ${ }^{137} \mathrm{Cs},{ }^{90} \mathrm{Sr}-{ }^{90} \mathrm{Y}$ & 35 \\
\hline
\end{tabular}

B-3 Radionucléides émetteurs $\alpha, \beta\left({ }^{239} \mathrm{Pu},{ }^{90} \mathrm{Sr}-{ }^{90} \mathrm{Y}\right)$, d'activité comprise entre 0,1 et $10 \mathrm{~Bq} . \mathrm{L}^{-1}$ en matrice de type eau de mer synthétique (salinité de l'ordre de 35 g. $\mathrm{L}^{-1}$ en présence de $\mathrm{NaHCO}_{3}$ ) acidifiée à l'acide nitrique $3 \mathrm{M}$;

C-3 Radionucléides émetteurs $\beta \gamma\left({ }^{137} \mathrm{Cs},{ }^{90} \mathrm{Sr}-{ }^{90} \mathrm{Y}\right)$ d'activité comprise entre 0,1 et 10 Bq.L $\mathrm{L}^{-1}$ en matrice de type eau de mer naturelle.

Note. La présence de plutonium dans les solutions nécessite une acidification relativement élevée.

Les données sont synthétisées dans le tableau 1.

\section{Détermination de la valeur de référence par radionucléide}

\subsection{Cas général}

Le Laboratoire National Henri Becquerel (LNHB), laboratoire primaire de métrologie, a fabriqué les solutions des 3 essais et pour chacune l'organisateur dispose d'une valeur de référence certifiée et de son incertitude pour l'activité de chacun des radionucléides ajouté.

\subsection{Cas particulier de la solution d'eau de mer réelle}

La solution C de l'essai $\mathrm{N}^{\circ} 3$ est élaborée par le laboratoire du GEA Cherbourg, à partir d'une eau de mer naturelle, dopée en radionucléides émetteurs béta. La concentration massique de sels est de 34 g.L. ${ }^{-1}$ dont 0,4 g.L $\mathrm{L}^{-1}$ en K et 3,3 $\mu$ g.L. ${ }^{-1}$ en uranium. La radioactivité $\mathrm{du}{ }^{40} \mathrm{~K}$ est déterminée de manière théorique par mesure de la concentration massique en potassium. Le calcul de l'activité bêta du ${ }^{40} \mathrm{~K}$ et de sa contribution dans l'évaluation de l'activité globale sont effectués selon une note de l'IRSN (2008). Les radionucléides artificiels rajoutés sont du ${ }^{137} \mathrm{Cs}$ d'activité égale à $0,88 \pm 0.05 \mathrm{~Bq} \cdot \mathrm{L}^{-1}$ et du ${ }^{90} \mathrm{Sr}$ d'activité égale à $0,95 \pm 0,05 \mathrm{~Bq} \cdot \mathrm{L}^{-1}$.

Le calcul théorique de l'activité bêta est donné par la relation suivante :

$$
A_{\text {bêta global }}=0,89 A_{40_{K}}+1,09 A_{137}{ }_{C s}+2 A{ }^{90} \mathrm{Sr}
$$

avec : $A_{40_{K}}$ activité volumique du potassium ${ }^{40} \mathrm{~K}, A_{137} C_{s}$ activité volumique du césium ${ }^{137} \mathrm{Cs}$ et $A_{9_{0} s_{r}}$ activité volumique du
Tableau 2. Valeurs de référence de la solution C3. Reference values - C3 solution.

\begin{tabular}{lccc}
\hline C3 (eau de mer naturelle) & Bq. $\mathrm{L}^{-1}$ & $\boldsymbol{u}(\boldsymbol{k}=\mathbf{2})$ & $\boldsymbol{U}_{\text {rel }}(\boldsymbol{k}=\mathbf{2})$ \\
\hline${ }^{90} \mathrm{Sr}$ et ${ }^{90} \mathrm{Y}$ & 1,9 & 0,1 & $5,7 \%$ \\
${ }^{137} \mathrm{Cs}$ & 0,94 & 0,04 & $4,3 \%$ \\
${ }^{40} \mathrm{~K}$ & 11,8 & 0,7 & $5,0 \%$ \\
Somme & 13,3 & 1,1 & $8,2 \%$ \\
\hline
\end{tabular}

strontium ${ }^{90} \mathrm{Sr}$. Les coefficients indiquent la contribution bêta dans l'activité totale :

- dans le cas du ${ }^{40} \mathrm{~K}$, émetteur $\beta \gamma$, seul $89 \%$ de l'activité globale est due aux émetteurs $\beta$;

- dans le cas du ${ }^{137} \mathrm{Cs}$, lors de l'émission de 85 photons $\gamma$ d'énergie $661 \mathrm{keV}$ il y a 109 électrons émis dont 9 électrons de conversion interne correspondant à la désintégration $\beta^{-}$;

- le ${ }^{90} \mathrm{Sr}$ et ${ }^{90} \mathrm{Y}$ sont émetteurs bêta à $100 \%$.

Les données de référence de la solution C3 sont données dans le tableau 2 .

\section{Organisation de l'essai et traitement statistique des résultats}

L'organisateur de l'essai utilise comme référentiel la norme NF ISO 17043 (ISO, 2010b) relative à l'organisation des comparaisons interlaboratoires. Il envoie les échantillons aux participants avec les consignes de réalisation des essais et un modèle de restitution des résultats qu'ils doivent renseigner le plus exhaustivement possible.

Pour chaque échantillon il est leur demandé d'effectuer cinq répliques indépendantes par type de détermination (radionucléide ou valeur de comptage), de fournir la moyenne de celles-ci assortie d'une incertitude à $k=2$ et de transmettre les résultats via une feuille de calcul au formalisme préétabli. À réception des résultats chaque participant reçoit une attestation avec un numéro qui n'est connu que de lui seul et de l'organisateur. Une fois recueilli l'ensemble des résultats la synthèse est réalisée grâce à la mise en œuvre d'un traitement statistique approprié.

La démarche générale consiste, à partir de tous les résultats parvenus :

- au calcul de la moyenne robuste,

- au calcul de l'écart type robuste, 
- à la détermination de l'incertitude robuste,

- à la détermination de l'incertitude robuste relative en $\%$.

Généralement, les tests statistiques utilisés dans le domaine de la mesure font référence à une population qui satisfait aux critères d'une loi normale (Dagnelie, 2011) définie par la moyenne et la variance. Cette hypothèse convient pour la majorité des cas rencontrés car les sources d'incertitude inhérentes à la mesure sont nombreuses, additives, de faible amplitude et indépendantes.

Cependant, la diversité des résultats obtenus et parfois le faible nombre de résultats à traiter avec une loi de distribution qui n'est pas toujours normale, nous incite à privilégier le recours aux méthodes robustes. Les méthodes robustes sont notamment basées sur l'emploi des rangs et sur la recherche de minimum autres que le minimum de la somme des carrés des écarts. Elles font appel à l'utilisation d'algorithmes, qui intègrent la totalité des résultats, y compris ceux considérés comme aberrants selon les critères d'une loi normale. Elles substituent la médiane (valeur centrale qui, après tri des données par ordre croissant, les sépare en 2 populations égales) à la moyenne.

Le traitement statistique mis en œuvre correspond à l'objectif de ces essais qui ne consistent pas à qualifier une méthode mais d'évaluer, de manière globale, l'aptitude des laboratoires à déterminer l'activité globale des radionucléides concernés.

\section{Calcul de la moyenne par essai et de l'incertitude robuste}

À chaque échantillon il est établi une moyenne calculée à partir des résultats unitaires correspondant aux répliques et au type de détermination.

$$
\overline{y_{i}}=\frac{1}{n_{i}} \sum_{j=1}^{n_{i}} y_{i j}
$$

$\overline{y_{i}}$ est la moyenne des répliques $j$ de la détermination $i, n_{i}$ est le nombre des répliques $j$ de la détermination $i$. Celle-ci est appelée «moyenne classique».

Le calcul de la moyenne robuste est effectué à l'aide de l'aide de l'algorithme A décrit dans les normes NF ISO 5725 (ISO, 1998) et NF ISO 13528 (ISO, 2005b). Elle est notée $x^{*}$ et l'écart type $s^{*}$. Dans les tableaux figurant dans le document, il est fait état de moyenne globale classique et robuste.

L'incertitude calculée selon les méthodes robustes est donnée par la relation suivante :

$$
u_{x}=\frac{1.25 \times s^{*}}{\sqrt{p}}
$$

$s^{*}$ étant défini comme l'écart type robuste des résultats calculés selon l'algorithme A, $p$ le nombre de laboratoires participants. La valeur assignée et son incertitude sont obtenues (Désenfant et Amarouche, 2007) à partir de la moyenne et de l'écart type robuste des résultats d'essais.

\section{Valeurs assignée des comptages}

Les valeurs d'activités alpha et bêta globales ne peuvent être certifiées puisque, par convention, le même rendement de mesure est affecté à chaque radionucléide alors que leur spectre d'énergie diffère.

Le LNHB certifie l'activité des radionucléides ajoutés sans pouvoir donner de valeur de référence pour les mesures d'activité alpha et bêta globales. Seules sont certifiées les valeurs d'activité des radionucléides émetteurs bêta ${ }^{90} \mathrm{Sr}-{ }^{90} \mathrm{Y},{ }^{137} \mathrm{Cs}$ et alpha ${ }^{239} \mathrm{Pu}$ contenus dans les solutions. Néanmoins il délivre, à titre indicatif, une valeur enveloppe représentative des émetteurs alpha et bêta présents dans la solution. En présence d'uranium, pour la détermination de l'activité bêta totale, les calculs tiennent compte de la présence des descendants à l'équilibre des radioéléments : ${ }^{234} \mathrm{Th},{ }^{234} \mathrm{~Pa}$ et ${ }^{231} \mathrm{Th}$.

La CETAMA a choisi de suivre les recommandations de la norme NF ISO 13528 (ISO, 2005b), qui préconise, dans le cas d'un échantillon dont il est difficile d'atteindre la valeur de référence par des méthodes métrologiques, de l'obtenir à partir du traitement statistique des résultats de comparaisons interlaboratoires. Cette valeur expérimentale aura le mérite d'être représentative d'un panel de laboratoires puisque les résultats rendus par les participants sont largement subordonnés aux conditions expérimentales propres à chacun.

Toutefois cette démarche présente la difficulté d'acquérir la preuve d'un réel consensus entre les participants qui mêlent, dans notre cas, des laboratoires experts à des laboratoires de contrôle et ne peut pas être établie. Par ailleurs il n'est pas possible de détecter un biais systématique susceptible d'altérer les résultats. Malgré ces limitations, cette démarche est souvent utilisée dans la détermination des traces dans les matrices naturelles. La discussion qui a eu lieu lors de la présentation des résultats a conclu à l'absence de biais avéré mais à une dispersion des résultats relativement élevée.

Les résultats satisfont à la condition de dispersion préconisée par le guide IUPAC (Thompson et al., 2006) : lors du calcul de l'incertitude. En effet il est nécessaire, pour valider la démarche, de s'assurer que l'écart type robuste calculé sur l'ensemble des résultats soit inférieur à 1,2 fois l'écart type de l'ensemble des résultats effectués de façon classique. Cet écart type est mentionné dans le document IUPAC sous la dénomination $\sigma_{p}$ «standard deviation for proficiency assessment ».

\section{Synthèse des résultats}

Le comptage alpha et bêta global est un moyen simple et efficace d'obtenir une vue d'ensemble radiologique. Cela permet de s'assurer que la radioactivité présente ne dépasse pas un certain seuil et, le cas échéant, d'orienter les investigations pour identifier l'origine des principaux radionucléides émetteurs. Ce mode de détermination comporte cependant certaines limites en termes d'exactitude et d'incertitudes associées.

La technique instrumentale le plus souvent utilisée est le compteur proportionnel à gaz. Il est basé sur le principe de l'ionisation d'un gaz conséquente à l'interaction avec le rayonnement alpha ou bêta émis par la source obtenue par évaporation de l'échantillon sur coupelle. Le système de détection est composé d'un détecteur placé sous haute tension dont le 
courant produit est proportionnel à l'activité de l'échantillon. La haute tension appliquée aux bornes des électrodes de la chambre d'ionisation permet de discriminer le signal en fonction de l'énergie d'émission.

L'activité globale est déterminée à partir du résultat de comptage de l'échantillon et du rendement de mesure déterminé avec le radionucléide de référence obtenu dans les mêmes conditions opératoires. Ce sont donc des mesures relatives qui s'effectuent par rapport à un étalon.

Une limitation est observée, notamment pour les radionucléides dont l'énergie bêta max est inférieure à $100 \mathrm{Kev}$ comme le ${ }^{63} \mathrm{Ni}, 1 \mathrm{le}^{210} \mathrm{~Pb}$, le ${ }^{228} \mathrm{Ra}$ et le ${ }^{241} \mathrm{Pu}$.

La mesure de la radioactivité d'un mélange de radioéléments dont la proportion, voire la nature, ne sont pas connues nécessite des investigations complémentaires en terme chimiques et radiologiques et un paramétrage adapté des instruments de mesure.

Les particules alphas, en raison de leur forte énergie de transfert linéique produisent un nombre élevé d'interactions avec le gaz dans le volume du détecteur. Une particule bêta produit beaucoup moins d'ions à travers son passage dans le gaz qu'une particule alpha.

Les résultats obtenus, en Bq.L ${ }^{-1}$, sont présentés dans les tableaux 3 et 4 .

Malgré le faible nombre de laboratoires ayant rendu leurs résultats, l'usage de statistiques robustes permet de calculer les indicateurs principaux (moyenne et écart type) et d'évaluer ceux-ci en tenant compte de cette contrainte.

\section{Synthèse}

\subsection{Source et étalonnage}

Puisque la mesure est basée sur la réalisation d'une source par dépôt d'une quantité de liquide sur une coupelle métallique suivi d'une évaporation il est important que l'étalonnage soit effectué dans des conditions géométriques rigoureusement identiques à celles de l'échantillon à mesurer.

Une attention particulière sera apportée pour s'assurer que, lors de la fabrication de la source à mesurer, la température d'évaporation ne soit pas supérieure à $85{ }^{\circ} \mathrm{C}$ pour éviter les projections ou les pertes de composés volatils. On sera tout particulièrement attentif à cette phase de préparation de l'échantillon en cas de présence réelle ou supposée de radionucléides volatils tels que le tritium, carbone, iode et technétium (EPA, 2009) pour les émetteurs béta, le ${ }^{210} \mathrm{Po}$ et le ${ }^{222} \mathrm{Rn}$ pour les émetteurs alphas. Dans tous les cas, l'épaisseur du dépôt doit être la plus fine et uniforme possible pour limiter les phénomènes d'auto absorption. Ceux-ci sont néanmoins corrigés pour la mesure alpha, en général à l'aide d'une courbe d'absorption.

Les rayonnements alpha et bêta ayant un faible pouvoir de pénétration dans la matière on observe une atténuation du flux qui est fonction de la densité de masse surfacique de la source. C'est pour cette raison que la source qui servira d'étalonnage doit respecter au mieux ces conditions.

Dans la très grande majorité des cas nous constatons que les résultats de mesure d'activité bêta globale estiment l'activité des radionucléides pris isolément en deçà de la valeur de référence. Nous traiterons séparément le cas des eaux naturelles chargées en sels de celui de l'eau de mer car les résultats ne présentent pas de fortes analogies.

Pour les eaux naturelles (hors eau de mer), sans conteste la charge saline accentue le phénomène de sous-estimation. La présence de radionucléides artificiels, qui sont souvent ceux choisis par les laboratoires pour étalonner leurs compteurs, a tendance à réduire l'écart. Les résultats obtenus sur les émetteurs alphas sont similaires même si l'on note que, pour l'eau ne contenant que le ${ }^{239} \mathrm{Pu}$, l'écart se réduit considérablement. À la réserve près que, pour la solution B-1 (solution « simple» du premier essai) cet écart avoisine les $20 \%$. Mise à part une activité faible par rapport aux autres essais qui a pour effet d'accentuer l'effet relatif, il n'existe pas d'interprétation satisfaisante.

Les résultats obtenus en faisant varier la nature du sel ne présentent pas de distorsion notable entre les résultats des activités alpha et bêta globales. On peut simplement envisager que l'hygroscopie (Sullivan et al., 2009) des sels de calcium et de sodium conduisent à des résultats dont la dispersion est sensiblement plus élevée que pour le sel de strontium. Ce phénomène influe directement sur la nature du dépôt par la modification de sa planéité et de la surface apparente. Ceci se traduit par une incertitude relative sensiblement plus importante notamment pour les alphas. Il est donc préconisé d'effectuer la mesure sitôt après la réalisation de la source et si nécessaire d'attendre quelques jours afin de s'affranchir de la présence de descendants $\mathrm{du}{ }^{222} \mathrm{Rn}$ qui peuvent générer un biais positif. Il est recommandé aux laboratoires d'être vigilant sur les conditions de conservation de la source notamment en ce qui concerne l'hygrométrie et de privilégier l'usage du dessiccateur.

Les résultats obtenus sur les eaux de mer sont tout à fait cohérents, qu'elles soient naturelles ou artificielles. Les écarts entre la valeur «enveloppe», somme de radionucléides et la valeur de comptage ne dépassent pas $5 \%$ en valeur absolue pour les activités bêta et $10 \%$ pour les activités alpha.

\subsection{Interprétation}

Le compteur proportionnel à gaz permet d'obtenir un mode de comptage alpha seul, bêta seul ou alpha bêta simultané. Parmi les causes possibles de variations, la sélection du mode de comptage peut générer des interférences des particules alphas avec les particules bêtas. La maitrise des interférences entre les émetteur $\alpha$ et $\beta$ nécessite de contrôler correctement le mode de comptage sélectionné. La diaphonie des voies $\alpha$ par rapport au bêta et inversement doit être vérifiée.

Les résultats de mesure des activités des émetteurs alpha ainsi que des émetteurs bêta d'énergie moyenne sont significativement plus faibles lorsque l'échantillon contient des matières solides dissoutes ou en suspension susceptibles de modifier sensiblement la nature du dépôt après évaporation sur coupelle. L'écart entre la valeur de référence et le comptage s'accroit en proportion de l'activité de la solution à mesurer, indépendamment des paramètres physico-chimiques. Pour des niveaux d'activité inférieurs à $10 \mathrm{~Bq} . \mathrm{L}^{-1}$, les variations sont inférieures à $20 \%$ et pour des niveaux supérieurs à 50 Bq.L $\mathrm{L}^{-1}$ (en incluant le ${ }^{40} \mathrm{~K}$ ), ces écarts sont majoritairement supérieurs 
Tableau 3. Données de valeurs de comptage béta en Bq.L $\mathrm{L}^{-1}$. Gross beta counting values (Bq.L $\left.\mathrm{L}^{-1}\right)$.

\begin{tabular}{lcccccc}
\hline $\begin{array}{l}\text { Numéro } \\
\text { d'échantillon }\end{array}$ & $\begin{array}{c}\text { Nombre de } \\
\text { laboratoires }\end{array}$ & $\begin{array}{c}\text { Valeur de } \\
\text { référence } \\
\text { activité RN }\end{array}$ & $\begin{array}{c}\text { Incertitude } \\
\text { sur la valeur } \\
\text { de référence }\end{array}$ & $\begin{array}{c}\text { Moyenne robuste } \\
\text { des comptages }\end{array}$ & $\begin{array}{c}\text { Incertitude sur } \\
\text { la moyenne } \\
\text { comptages }\end{array}$ & $\begin{array}{c}\text { Ecart relatif entre la } \\
\text { moyenne des comptages et } \\
\text { la valeur de référence RN }\end{array}$ \\
\hline A-1 & 14 & 79,28 & 2,70 & 61,05 & 2,50 & $-23 \%$ \\
\hline B-1 & 13 & 2,09 & 0,02 & 2,15 & 0,10 & $3 \%$ \\
C-1 & 13 & 93,26 & 2,96 & 76,67 & 3,40 & $-18 \%$ \\
D-1 & 13 & 93,04 & 2,96 & 69,38 & 2,89 & $-25 \%$ \\
A-2 & 16 & 7,13 & 0,05 & 5,75 & 0,36 & $-19 \%$ \\
\hline B-2 & 17 & 6,92 & 0,08 & 5,85 & 0,26 & $-15 \%$ \\
C-2 & 16 & 9,40 & 0,09 & 8,48 & 0,43 & $-10 \%$ \\
A-3 & 34 & 3,75 & 0,03 & 3,42 & 0,04 & $-3 \%$ \\
\hline B-3 & 13 & 15,7 & 0,03 & 15,19 & 0,53 & $4 \%$ \\
\hline C-3 & 13 & 13,3 & 1,1 & 13,88 & 0,46 & \\
\hline
\end{tabular}

Tableau 4. Données de valeurs de comptage alpha en Bq.L $\mathrm{L}^{-1}$.

Gross alpha counting values (Bq. $\left.\mathrm{L}^{-1}\right)$.

\begin{tabular}{lcccccc}
\hline $\begin{array}{l}\text { Numéro } \\
\text { d'échantillon }\end{array}$ & $\begin{array}{c}\text { Nombre de } \\
\text { laboratoires }\end{array}$ & $\begin{array}{c}\text { Valeur de } \\
\text { référence } \\
\text { activité RN }\end{array}$ & $\begin{array}{c}\text { Incertitude } \\
\text { sur la valeur } \\
\text { de référence }\end{array}$ & $\begin{array}{c}\text { Moyenne robuste } \\
\text { des comptages }\end{array}$ & $\begin{array}{c}\text { Incertitude sur } \\
\text { la moyenne } \\
\text { comptages }\end{array}$ & $\begin{array}{c}\text { Ecart relatif entre la } \\
\text { moyenne des comptages et } \\
\text { la valeur de référence RN }\end{array}$ \\
\hline A-1 & 15 & 72,36 & 2,20 & 55,68 & 2,00 & $-23 \%$ \\
B-1 & 14 & 1,05 & 0,01 & 1,24 & 0,08 & $18 \%$ \\
C-1 & 14 & 78,67 & 2,21 & 63,72 & 3,20 & $-19 \%$ \\
D-1 & 14 & 79,02 & 2,21 & 56,68 & 4,34 & $-28 \%$ \\
A-2 & 15 & 2,07 & 0,02 & 1,65 & 0,19 & $-20 \%$ \\
B-2 & 16 & 2,07 & 0,02 & 1,94 & 0,10 & $-19 \%$ \\
C-2 & 15 & 2,07 & 0,02 & 1,68 & 0,11 & $-2 \%$ \\
\hline A-3 & 16 & 1,93 & 0,03 & 1,89 & 0,14 & $8 \%$ \\
\hline B-3 & 6 & 1,58 & 0,03 & 1,71 & 0,37 & \\
\hline
\end{tabular}

à $20 \%$. Un premier constat montre une tendance qui semble intensifier cet écart avec l'activité de la solution à mesurer. Il est recommandé d'effectuer l'étalonnage du compteur dans des conditions les proches possibles des échantillons à mesurer (niveau d'activité, radionucléide émetteur, charge saline...).

\section{Conclusion}

Pour les circuits présentés, la détermination de la valeur de consensuelle ou assignée est obtenue à partir de la moyenne robuste des résultats des laboratoires participants. Cette méthode itérative est particulièrement intéressante parce qu'elle minimise l'influence des résultats extrêmes et qu'elle reste cohérente lorsqu' on ne dispose que d'un petit nombre de données.

Un écart significatif entre la valeur enveloppe déterminée par la somme des activités des radionucléides pris isolément et la moyenne des résultats de comptage des laboratoires est observée sur l'ensemble résultats. L'analyse de ceux-ci conduit à considérer que comptage d'une solution dont l'activité est inférieure à $10 \mathrm{~Bq} . \mathrm{L}^{-1}$ conduit à une valeur de mesure qui sousestime d'environ 10 à $20 \%$ la somme de l'activité des radionucléides présents. Ce phénomène, par ailleurs connu et identifié a pu être quantifié. Il est sensiblement plus marqué pour les alphas et s'accentue lorsque l'activité de l'échantillon augmente. L'influence de la charge saline sur la dispersion des résultats et donc sur l'incertitude associée est indéniable. D'une manière générale, cette dispersion, sensiblement plus élevée pour les alphas, n'excède pas $10 \%$ indépendamment de la nature du sel.

L'examen des résultats révèle un caractère inattendu sur la mesure bêta d'un échantillon «idéal » c'est-à-dire une eau de type naturelle, relativement peu chargée en sel, ne contenant que des radionucléides artificiels à faible niveau d'activité. Dans un cas on observe une surestimation des émetteurs bêta et dans l'autre une sous-estimation. Ceci peut être lié à l'usage d'un coefficient d'auto-absorption censé compenser la perte du rayonnement lié à la composition saline de la solution. On peut supposer que, dans le cas d'une charge saline inférieure au g.L $\mathrm{L}^{-1}$, l' application d'un coefficient trop élevé (ordinairement appliqué à une solution plus chargée) peut conduire à une surestimation de l'activité. Dans le cas des activités alpha, on observe, sur ce type de solution, un comportement davantage conforme à ceux attendus avec un écart inférieur à $5 \%$ par rapport à l'activité réellement présente.

Les résultats sur l'eau de mer sont très cohérents et traduisent une bonne maitrise du processus de préparation de l'échantillon et de la mesure incluant l'usage des coefficients d'auto-absorption. On peut simplement relever que la cause de l'écart observé sur l'eau de mer naturelle, dopée en émetteurs bêta et gamma, peut être due à la combinaison d'émetteurs bêta et gamma dont l'énergie d'émission est sensiblement différente. 


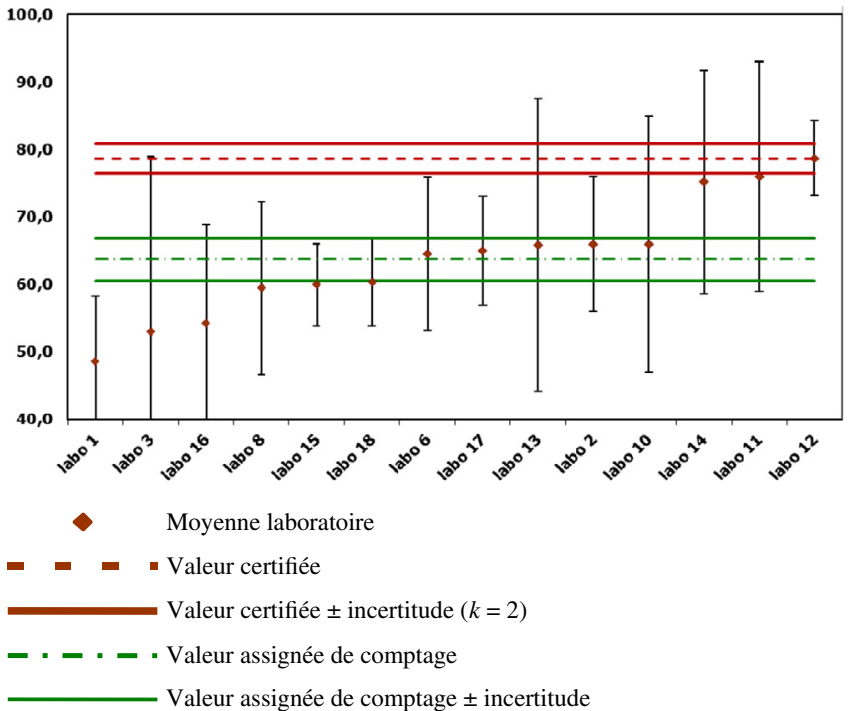

Figure 1. Valeur d'activité alpha de la solution C-1 en Bq. $\mathrm{L}^{-1}$. Gross alpha solution C-1.

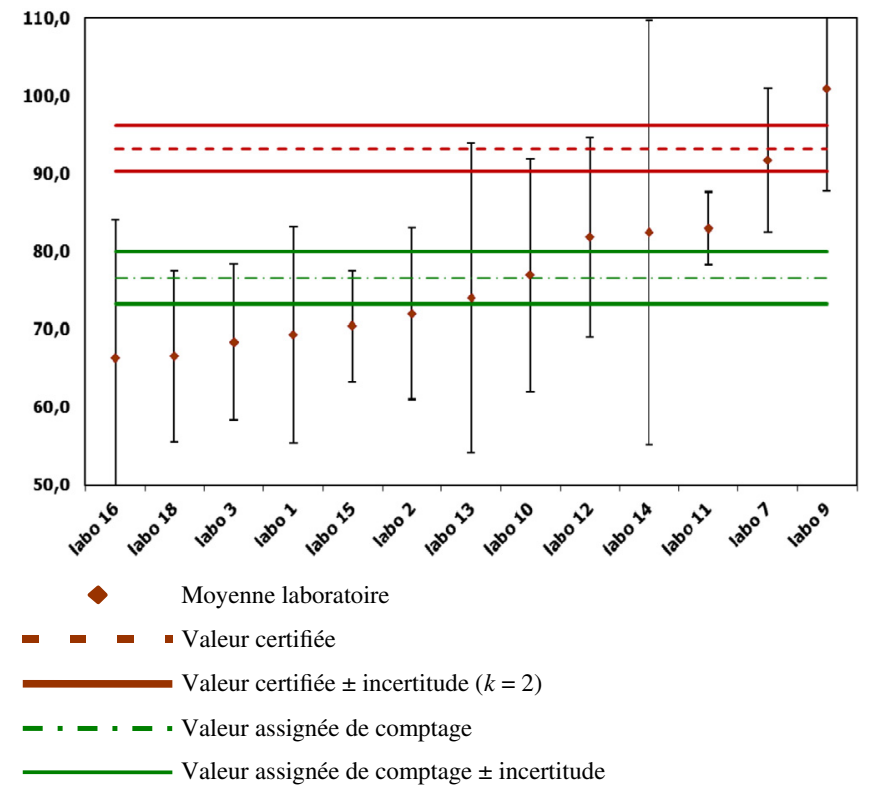

Figure 2. Valeur d'activité bêta de la solution C-1 en Bq.L ${ }^{-1}$. Gross beta solution C-1.

D'une manière générale, dès que l'on s'éloigne des conditions que l'on peut qualifier d'optimales (proche des conditions de l'étalonnage) et que l'on tend vers une meilleure représentativité de la matrice environnementale, on constate un écart entre la somme des activités et la valeur de comptage sensiblement plus élevée, tout en restant dans des limites qui n'excèdent pas $20 \%$.

Le niveau d'activité de la solution est un facteur prépondérant pour la qualité du résultat. Il faut cependant noter que les 3 essais se sont déroulés sur une période de 4 ans. Pour chacun d'entre eux un cahier des charges a été élaboré à partir d'un besoin défini par l'ensemble des laboratoires participants à la CETAMA. Chacun a donné lieu à une synthèse et à une évaluation réalisée lors de réunions. Le choix des ma-

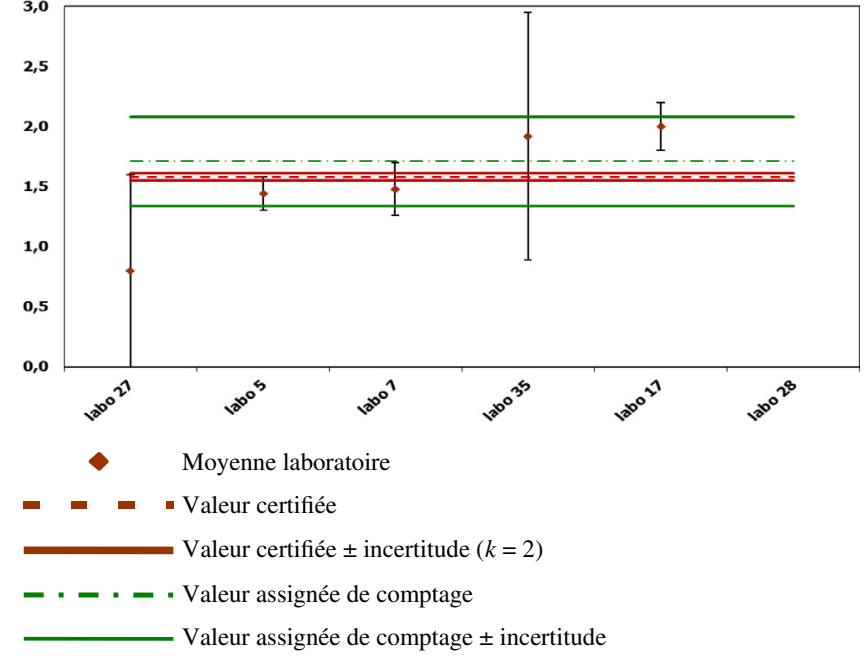

Figure 3. Valeur d'activité alpha de la solution B-3 en Bq.L $\mathrm{L}^{-1}$. Gross alpha solution B-3.

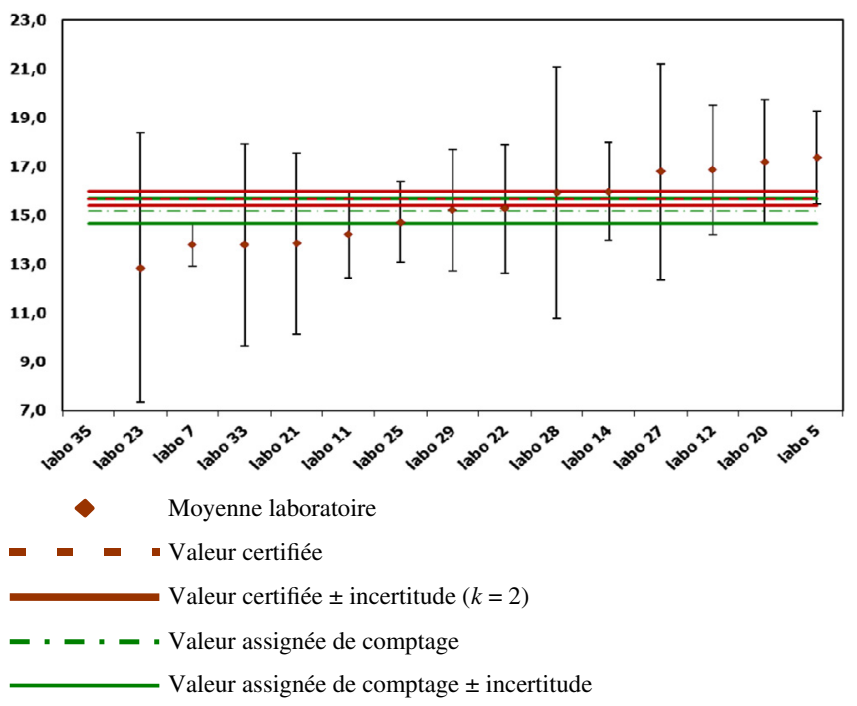

Figure 4. Valeur d'activité bêta de la solution B-3 en Bq.L. ${ }^{-1}$. Gross beta solution B-3.

trices, des caractéristiques et des radionucléides a fait l'objet d'un consensus obtenu par l'ensemble des membres. Ce travail d'optimisation effectué par les laboratoires, au travers d'un partage de connaissance, s'est traduit par une amélioration des performances et la rédaction d'une méthode de mesurage des activités alpha globale et bêta globale des eaux salines ${ }^{9}$. L'obtention de résultats en progrès lors des circuits successifs en est le révélateur. Cet exercice atteste la conformité de la démarche qui permet d'obtenir une valeur de référence et de son incertitude à partir de résultats expérimentaux obtenus par comparaison interlaboratoires. On pourra toujours la comparer, le cas échéant, à une valeur de référence certifiée qui ne peut être obtenue que sur chacun des radionucléides composant la solution. En respect des exigences règlementaires cette caractérisation de premier niveau permet de contrôler la présence de radionucléides émetteurs alpha ou bêta dans les eaux naturelles.

${ }^{9}$ Méthode CETAMA (en cours de publication). 
À ce niveau d'activité les écarts observés lors de la synthèse des essais montrent qu'ils sont compatibles avec les objectifs de cette caractérisation.

Remerciements. Nous remercions tous les participants des différents essais organisé par la CETAMA ainsi que les membres du GT 18 « analyse de l'eau » qui, par leur contribution à la synthèse des résultats ont permis à ce travail d'aboutir.

\section{Références}

ASN (2008) Annexe à la Décision N²008-DC-0099 de l'Autorité de sûreté nucléaire du 29 avril 2008 portant organisation du réseau national de mesures de la radioactivité de l'environnement et fixant les modalités d'agrément des laboratoires.

ASN (2015) Décision N²015-DC-0500 de l'Autorité de sûreté nucléaire du 26 février 2015 portant modification de la décision $\mathrm{N}^{\circ}$ 2008-DC-0099 de l'Autorité de sûreté nucléaire du 29 avril 2008 relative à l'organisation du réseau national de mesures de la radioactivité de l'environnement et fixant les modalités d'agrément des laboratoires.

Bizet J., Boog F. (2014) Rapport d’information du sénat N55 enregistré le 21 mai 2014.

Dagnelie P. (2011) Statistique théorique et appliquée - Tome 2. Inférence statistique à 1 et 2 dimensions (De Boeck, Bruxelles).

Désenfant S., Amarouche S. (2007) Evaluation d'aptitude par comparaison interlaboratoires. LNE Publication $13^{\mathrm{e}}$ congrès métrologie.

EPA (2009) Technical Notes for EPA Method 900.0 Gross Alpha and Gross Beta Radioactivity in Drinking Water.
EURATOM (2013) Directive 2013/51/EURATOM du conseil du 22 octobre 2013 fixant des exigences pour la protection de la santé de la population en ce qui concerne les substances radioactives dans les eaux destinées à la consommation humaine.

IRSN (2008) Calcul de la contribution de ${ }^{40} \mathrm{~K}$ à l'activité bêta global. Note IRSN/DEI/STEME 2008-04.

ISO (1998) NF ISO 5725-5. Exactitude (justesse et fidélité) des résultats et méthodes de mesure. Partie 5 : Méthodes alternatives pour la détermination de la fidélité d'une méthode de mesure normalisée.

ISO (2005a) NF EN ISO/CEI 17025. Exigences générales concernant la compétence des laboratoires d'étalonnages et d'essais.

ISO (2005b) PR NF ISO 13528 Méthodes statistiques utilisées dans les essais d'aptitude par comparaisons interlaboratoires.

ISO (2010a) NF ISO 10704. Qualité de l'eau - Mesurage des activités alpha globale et bêta globale des eaux non salines - Méthode par dépôt d'une source fine.

ISO (2010b) Norme NF EN ISO/CEI 17043 - Évaluation de la conformité, exigences générales concernant les essais d'aptitude.

Ministère de la santé (2007) Circulaire Nº DGS/EA4/2007/232 du 13 juin 2007 relative au contrôle et à la gestion du risque sanitaire liés à la présence de radionucléides dans les eaux destinées à la consommation humaine, à l'exception des eaux conditionnées et des eaux minérales naturelles.

Sullivan R.C. et al. (2009) Effect of chemical mixing state on the hygroscopicity and cloud nucleation properties of calcium mineral dust particles, Atmos. Chem. Phys. 9, 3303-3316.

Thompson M., Ellison S.L.R., Wood R. (2006) The International Harmonized Protocol for the proficiency testing of analytical chemistry laboratories, IUPAC Technical Report, Pure Appl. Chem. 78 (1), 145-196.

WHO (2011) Guidelines for drinking-water quality, $4^{\mathrm{e}}$ edn.

Cite this article as: G. Granier, P. Nardoux, D. Roudil. Comparaisons interlaboratoires de comptage de l'activité des émetteurs alpha et bêta dans des eaux à différentes charges salines. Radioprotection 50(3), 215-223 (2015). 